\title{
The Relation between Preference for Predictability and Autistic Traits
}

Accepted for publication in Autism Research

\author{
Judith Goris $^{1}$, Marcel Brass ${ }^{1}$, Charlotte Cambier, Jeroen Delplanque ${ }^{3}$, Jan R. Wiersema ${ }^{2}, \&$ \\ Senne Braem ${ }^{1,3}$ \\ ${ }^{1}$ Department of Experimental Psychology, Ghent University, Henri Dunantlaan 2, 9000 Ghent, \\ Belgium \\ ${ }^{2}$ Department of Experimental Clinical and Health Psychology, Ghent University, Henri \\ Dunantlaan 2, 9000 Ghent, Belgium \\ ${ }^{3}$ Department of Psychology, Vrije Universiteit Brussel, Pleinlaan 2, 1050 Brussel
}

Short title: Preference for Predictability in Autism

\section{Correspondence:}

Judith Goris

Department of Experimental Psychology

Henri Dunantlaan 2

B - 9000 Ghent

BELGIUM

E-mail: judith.goris@ugent.be

This is the pre-peer reviewed version of the following article: Goris, J., Brass, M., Cambier, C., Delplanque, J., Wiersema, J.R., \& Braem, S. (In press). The Relation between Preference for Predictability and Autistic Traits. Autism Research, which will be published in final form at https://doi.org/10.1002/aur.2244. This article may be used for non-commercial purposes in accordance with Wiley Terms and Conditions for Use of Self-Archived Versions. 


\section{Acknowledgements}

J.G. was supported by a PhD fellowship by the FWO - Research Foundation Flanders. M.B. was supported by a BOF-ZAP grant of Ghent University. The authors declare no conflict of interest. 


\begin{abstract}
A common idea about individuals with autism spectrum disorder (ASD) is that they have an above average preference for predictability and sameness. However, surprisingly little research has gone towards this core symptom, and some studies suggest the preference for predictability in ASD might be less general than commonly assumed. Here, we investigated this important symptom of ASD using three different paradigms, which allowed us to measure preference for predictability under well-controlled experimental conditions. Specifically, we used a dimensional approach by investigating correlations between autistic traits (as measured with the Autism Spectrum Quotient and Social Responsiveness Scale in a neurotypical population) and the scores on three different tasks. The 'music preference' task assessed preferences for tone sequences that varied in predictability. The 'perceptual fluency' task required participants to evaluate stimuli that were preceded by a similar versus dissimilar subliminally presented prime. The 'gambling' task presented four decks of cards that had equal outcome probabilities, but varied in predictability. We observed positive correlations between autistic traits and a preference for predictability in both the music preference and perceptual fluency task. We did not find our hypothesized correlation with gambling behavior, but did observe a post-hoc correlation showing that participants high on autistic traits were faster to choose the predictable deck. Together, these findings show that a relation between autistic traits and preference for predictability can be observed in a standardized lab environment, and should be considered an important first step towards a better, more mechanistic understanding of insistence on sameness in ASD.
\end{abstract}

Keywords: autism; ASD; preference; predictability; insistence on sameness 


\section{Lay summary}

A core symptom of autism spectrum disorder (ASD) is a strong preference for predictability, but little research has gone towards it. We show that neurotypical adults with more autistic traits have stronger preferences for predictable tunes, evaluate images that can be predicted as more beautiful, and are faster in choosing a gambling option resulting in predictable reward. These results offer first important evidence that insistence on sameness in ASD can be studied in controlled lab settings. 


\section{Introduction}

One of the main symptoms of autism spectrum disorder (ASD) is an above average preference for predictability and sameness. In the DSM-5, this insistence on sameness is exemplified as "extreme distress at small changes, difficulties with transitions, rigid thinking patterns, greeting rituals, need to take same route or eat same food every day" (American Psychiatric Association, 2013, p. 50). It has been suggested that this insistence drives other symptoms of ASD such as sensory sensitivities (Neil, Olsson, \& Pellicano, 2016) and repetitive behaviors like motor stereotypies (Wigham, Rodgers, South, McConachie, \& Freeston, 2015). In fact, recent theories propose that a deficit in dealing with unexpected information could be the single impairment underlying all ASD symptoms (Lawson, Rees, \& Friston, 2014; Van de Cruys et al., 2014). However, the precise mechanisms underlying these preferences in ASD received surprisingly little attention in research.

In the neurotypical population, several studies have shown that humans prefer stimuli with a certain amount of predictability. For example, it has been shown that participants prefer stimuli that were presented in a predictable configuration over stimuli from random configurations (Trapp, Shenhav, Bitzer, \& Bar, 2015). Also in visual search paradigms, participants seem to like targets more that are predictable in color (Chetverikov \& Kristjansson, 2015), or configurations that are predictive of target location (Ogawa \& Watanabe, 2011). Similarly, in cognitive control paradigms, it has been shown that people automatically evaluate stimuli as more positive, when they predict the relevant task dimension, relative to stimuli that do not (Dreisbach \& Fischer, 2012; Fritz \& Dreisbach, 2013; Schouppe et al., 2015). This effect on preferences is even found when the (un)predictability of stimuli is manipulated subliminally, as stimuli that are preceded subliminally by their own contours are liked better than when they 
were preceded by contours of a different picture (Forster, Leder, \& Ansorge, 2012; Westerman, Lanska, \& Olds, 2015). Last, a substantial literature on the mere exposure effect has demonstrated that stimuli that are more familiar to people (e.g., after being exposed to them more) are typically preferred over stimuli that are less familiar (Zajonc, 2001).

Surprisingly, however, despite this abundance of studies on a general preference for predictability, there has been little to no research on this in the context of ASD. As far as we know, this core symptom of ASD has not yet been captured under controlled experimental conditions. Some studies from related literatures have hinted at a stronger preference for sameness in ASD. For example, some have shown a stronger tendency to repeat the same task (Poljac, Poljac, \& Yeung, 2012), more perseveration errors when task rules change (Landry \& Al-Taie, 2016), and reduced risk-taking behavior (South et al., 2014). However, these studies were not directly set up to investigate insistence on sameness and therefore the manipulation of other variables might have confounded the study of preference for predictability. Notably, recent theories grounded in predictive coding proposed that a problem in dealing with unexpected information could actually be the core deficit that can account for all ASD symptoms (Lawson et al., 2014; Palmer, Lawson, \& Hohwy, 2017; Van de Cruys et al., 2014). Specifically, these theories suggest that there could be a deficit in the context-sensitive adjustment to prediction errors in ASD, meaning that individuals with ASD have difficulties with estimating in which context a surprising event is important and in which it is not. Recent studies also found evidence for this hypothesis (Goris et al., 2018; Lawson, Mathys, \& Rees, 2017). More generally, these theories emphasize this role of (aberrant) predictive mechanisms in explaining brain and behavior, and clearly call for more systematic research on this insistence on sameness in ASD. 
In a recent study, we were the first to study the relation between preference for predictability and ASD in a controlled lab environment (Goris, Deschrijver, Trapp, Brass, \& Braem, 2017). Specifically, we measured preferences for stimuli that were presented earlier in either a fixed, predictable configuration or a random configuration. While we replicated the previous finding that people show a preference for stimuli from fixed configurations (Trapp et al., 2015), this preference did not correlate with autistic traits in a neurotypical population. These results of course cannot refute a general preference for predictability in ASD, but do suggest that these preferences might be less general than first thought, or restricted to more complex situations (e.g. that require purposive action or in which participants are unaware of the predictability manipulation). Notably, however, there was a clear distinction in our paradigm between the 'learning phase', where subjects could learn about the predictive nature of the stimuli, and the subsequent 'preference phase', where participants had to indicate their preference (Goris et al., 2017). Therefore, it is possible that participants with more autistic traits did experience a stronger preference for the fixed configurations, but were less likely to transfer these preferences from the learning phase, to the subsequent preference phase. This would be consistent with the suggestion that individuals with ASD are more likely to experience different contexts anew (Van de Cruys et al., 2014), and thus less likely to transfer their preferences from one context to another.

In the current study, we wanted to investigate the relation between preference for predictability and autistic traits more in depth, and tried to remedy these previous limitations by using paradigms where the preference for predictability was measured in the task itself. In fact, to further ensure we approached this endeavor from multiple angles, we used three different paradigms measuring preference for predictability. First, in the Music Preference paradigm (as 
developed by Delplanque et al., 2019), participants listened to specific tone sequences varying in predictability, followed by a forced-choice preference judgment task. Earlier findings show an inverted U-shape pattern, indicating strongest preferences for stimuli with medium complexity (Delplanque, De Loof, Janssens, \& Verguts, 2019). Second, we used a Perceptual Fluency paradigm in which predictability was manipulated subliminally. Participants were presented with images that were preceded either by a subliminal presentation of their own contours (congruent), or by contours from a different image (incongruent), and rated each image on a 7point Likert scale. It has been shown that participants show higher liking ratings for congruent than for incongruent images in this paradigm (Forster et al., 2012; Reber, Winkielman, \& Schwarz, 1998; Westerman et al., 2015). Last, we developed a new (Equal Options) Gambling paradigm similar to the Iowa Gambling Task (IGT) (Bechara, Damasio, Damasio, \& Anderson, 1994; Bechara, Damasio, Tranel, \& Damasio, 2005), in which participants are instructed to win as much money as possible, by choosing a card out of four decks associated to different outcomes varying in predictability. However, unlike the IGT, outcome predictability was the only manipulated variable, and thus the average expected reward outcome was the same for each deck. Importantly, in all three of these paradigms, preference judgments (in the Music Preference and Perceptual Fluency task) or choice behavior (in the Gambling task) followed immediately after stimulus presentation, tackling the possible problem of transferring preferences from one phase to another (Goris et al., 2017). Furthermore, participants were not made aware of the predictability manipulation, unlike our previous study where participants were explicitly instructed to categorize the predictable versus unpredictable configurations in the learning task.

We employed a dimensional approach using questionnaires measuring autistic traits in a large neurotypical sample of 164 participants, in order to estimate correlations between autistic 
traits and preference for predictability. ASD is considered a clinical condition distinct from the neurotypical population, but it is suggested that autistic traits are continuously distributed across the general population (Constantino \& Todd, 2003). Many earlier studies in the neurotypical population have shown that a correlational approach can produce relevant insights about ASD (Grinter et al., 2009; Robertson \& Simmons, 2013; Stewart, Watson, Allcock, \& Yaqoob, 2009; Walter, Dassonville, \& Bochsler, 2009). Furthermore, by using this diverse set of tasks and questionnaires with different subscales, we could also explore whether the preference for predictability in ASD is a generalizable trait underlying behavior in a variety of tasks, or whether different autistic traits are related to different exhibitions of preference for predictability in different contexts. In case of the former, we can expect strong relations between preferences in the different paradigms (for a similar approach, see Tulver, Aru, Rutiku, \& Bachmann, 2019), which we investigated with pairwise correlations. 


\section{Methods}

All data, experiment files and analysis scripts can be found on the open science framework (https://osf.io/pkq3u).

\section{Participants}

An a-priori power analysis showed that to detect a Pearson's r correlation of .20 or higher with a one-tailed significance test at $p=.05$ and a power of $80 \%$, a sample size of 153 participants is needed. We recruited 164 participants from the Ghent University population. Three participants were removed from the analysis because they had inconsistent questionnaire scores (see below). Thus, the final sample consisted of 161 participants (114 female, 135 right-handed). Their age ranged from 18 to 50 years $(M=22, S D=4.8)$. Fifty-one participants received a course credit in return for their participation. The other 110 participants were paid $€ 10$. Sample sizes for the correlational analyses are 160 for the AQ and 157 for the SRS-A, due to missing data in the questionnaires. In addition, we had to exclude five more participants in the Music Preference paradigm, due to a technical problem during data collection, and nine participants were removed in the Gambling paradigm because they didn't choose each deck at least five times (see below).

\section{Procedure}

Participants first conducted the Music Preference task $( \pm 35 \mathrm{~min})$, followed by the Perceptual Fluency task $( \pm 8 \mathrm{~min})$, the Gambling task $( \pm 7 \mathrm{~min})$ and the questionnaires $( \pm 10$ $\min )$. 


\section{Questionnaires}

Two ASD questionnaires were used: the Autism-Spectrum Quotient (AQ) and the Social Responsiveness Scale - Adult version (SRS-A). Both were self-report adult versions in Dutch.

Autism-spectrum quotient. The AQ is one of the most well-known questionnaires for measuring autistic traits (Baron-Cohen et al., 2001; Dutch version: Hoekstra, Bartels, Cath \& Boomsma, 2008). It consists of five subscales: communication, social skills, imagination, attention to detail, and attention switching. Each subscale has 10 items, resulting in a 50-item questionnaire. The items are answered on a 4-point Likert scale (“definitely agree", "slightly agree", "slightly disagree", "definitely disagree"). While the AQ cannot be used as a diagnostic instrument (e.g. Ashwood et al., 2016), it does show good concurrent validity with other measures of autistic traits (Ingersoll, Hopwood, Wainer, \& Brent Donnellan, 2011; White, Ollendick, \& Bray, 2011).

Social Responsiveness Scale-Adult version. The SRS-A is a questionnaire that measures social symptoms of ASD (Constantino et al., 2003; Dutch version: Noens, De la Marche \& Scholte, 2012). It consists of four subscales: social awareness, social communication, social motivation, and rigidity and repetitiveness. The 65 items are answered on a 4-point Likert scale ("never true", "sometimes true", "often true", "always true").

Inconsistent scores. The scale used in the AQ is opposite from that in the SRS-A, meaning that a score of "1" in AQ means "definitely agree", while it means "never true" in the SRS-A. As a few participants had a high total score on one questionnaire but a low score on the other, we suspected some might have used the scales in a wrong way. To identify these participants, we selected eight questions that were highly similar in both questionnaires and 
compared responses to these questions. Three participants were excluded because they had a difference of two or more points on at least five of these questions.

\section{Experimental tasks}

Music Preference paradigm. In this paradigm adapted from Delplanque and colleagues (2019), participants were presented with two sequences of seven tones on each trial and had to indicate which of the two they preferred. The sequences varied in unpredictability or entropy. Seven tones played on a grand piano were used to compose the sequences: F3, G3, A3, B3, C4, D4 and E4 (or fa, sol, la, si, do, re, mi).

For all possible sequences consisting of these tones, we calculated Shannon entropy for the probability of each unique tone (first-order entropy) and for the probability of each tone transition (second-order entropy) using the following formula (see also, Delplanque et al., 2019):

$$
\text { Entropy }=-\sum_{i=1}^{n} p\left(x_{i}\right) \log p\left(x_{i}\right)
$$

For example, in the sequence A3, A3, B3, B3, B3, B3, B3, the probability of the tone A3 is $2 / 7$, while $\mathrm{B} 3$ has a probability of $5 / 7$. Therefore, first-order entropy is calculated as the negative of the sum of $(2 / 7) * \log (2 / 7)$ and $(5 / 7) * \log (5 / 7)$, which is 0.60 . Regarding the tone transitions, there is a probability of $5 / 6$ for a repetition (i.e. subsequent tones were identical) and a probability of 1/6 for moving up one tone on the scale mentioned above (A3 to B3). Thus, second-order entropy is calculated as the negative of the sum of $(5 / 6) * \log (5 / 6)$ and $(1 / 6) * \log$ $(1 / 6)$, which is 0.45 . We defined entropy here as the average of first-order and second-order entropy, which is 0.53 in the example sequence. However, the results were similar when analyzed on first-order or second-order entropy only. 
We divided all possible sequences consisting of the seven tones in 11 entropy classes: $0.31-0.45,0.46-0.60 \ldots$ until $1.66-1.80$ and $>1.81$. Sequences with entropy lower than 0.31 were not included since there were only 7 of them. Out of all possible sequences, we then randomly selected 20 sequences of each class that were presented to all participants. Every stimulus sequence was only presented once. Each entropy class was compared with every other entropy class twice, once in the first position and once in the second position (see below). Thus, each participant was presented with 220 trials.

As illustrated on Figure 1A, participants first saw a fixation cross for $1000 \mathrm{~ms}$, after which the number " 1 " was presented on screen while the first tone sequence was presented auditorily. Each tone lasted for $350 \mathrm{~ms}$ and inter-tone-interval was $150 \mathrm{~ms}$, resulting in sequences of $3350 \mathrm{~ms}$. This was followed by a screen showing "End of the first sequence" (in Dutch). Next, the number " 2 " was presented while the second tone sequence played for $3350 \mathrm{~ms}$. This was followed by the visual question: "Which sequence do you prefer?" (in Dutch). Participants were instructed to answer with the buttons A for the first sequence and $\mathrm{P}$ for the second sequence on an azerty computer keyboard.

Perceptual Fluency paradigm. In this paradigm similar to Forster, Leder and Ansorge (2012), participants had to indicate their preference for congruent and incongruent images. 150 gray-scale stimuli were selected from the set provided by Rossion and Pourtois (2004). Of those, 100 images were selected as target images, and the remaining 50 were used to create incongruent primes. Trials could be either congruent, in which the target was preceded by a prime consisting of the target's contours (created with the Difference of Gaussians method for edge detection in GIMP 2), or incongruent, when the target was preceded by a prime consisting of contours of a 
different image. The experiment consisted of 50 congruent and 50 incongruent trials, which were presented in a random order.

Each trial started with a fixation cross of $1000 \mathrm{~ms}$, followed by a mask which was presented for $500 \mathrm{~ms}$, a prime for $33.33 \mathrm{~ms}$, and another mask for $500 \mathrm{~ms}$ (see Figure 2A). Next, the target was presented for $2000 \mathrm{~ms}$ and followed by the question "How beautiful was this image according to you?" (in Dutch), which had to be answered on a 7-point Likert scale using the mouse, without time constraint. Trials with reaction times (RT) higher than three standard deviations (SD) from the individual means were removed from analyses.

Gambling paradigm. Last, we developed a gambling task in which participants were instructed to win as much money as possible (see Figure 3A). The five participants with the highest scores received a shopping coupon of $€ 30$. On each trial, participants saw four decks of cards, labeled A, B, C and D. Participants chose one of these decks by clicking on it, without time constraint. Next, the amount of money they won appeared on the screen for $1500 \mathrm{~ms}$. The total amount of money collected was continuously presented at the top of the screen. 200 trials were presented.

Unknown to the participants, each of the four decks had an average reward outcome of $€$ 250. Only the predictability of the gain was manipulated. The fixed deck always returned a gain of $€ 250$. The three other decks returned a gain drawn from a normal distribution with mean $€$ 250 and SDs 10, 30 and 70. The order of decks was randomized across participants (but remained fixed over the course of the experiment). Participants that did not choose each deck at least five times were excluded from analyses, which were nine participants. Trials with RTs higher than three SDs from the individual means were removed from analyses. 


\section{Data analysis}

Because we did not have differential hypotheses for the SRS-A versus AQ, we ran an unrotated Principal Components Analysis (PCA) on the total scores of the AQ and SRS-A. This way, we could extract a single factor for autism traits, allowing us to simplify the interpretation of the correlational analysis. This factor explained $84 \%$ of the total variance of the AQ and SRSA scores. In what follows, we will always report the correlations with the PCA factor first, followed by the correlations with AQ and SRS-A separately. We used Spearman's rank-ordered correlation coefficients $\rho$ to detect correlations, because these are less sensitive to outliers. Although our clear unidirectional hypotheses allowed for one-tailed hypothesis testing, we report two-tailed $p$ values for ease of interpretation. Similarly, we would like to note that the main correlations with the PCA factor across the three paradigms survived a Holm-Bonferroni correction for multiple comparisons (on our one-tailed, predicted correlations), but we report the uncorrected $p$ values for ease of interpretation. 


\section{Results}

\section{Questionnaires}

AQ total scores ranged from 70 to $152(M=104.41, S D=14.47)$, using dimensional scoring. With binary scoring, the total scores ranged from 4 to $38(M=15.74, S D=6.55) .6$ participants (4\%) scored between 26 and 32, which might suggest mild ASD (Woodbury-Smith, Robinson, Wheelwright, \& Baron-Cohen, 2005). Six participants (4\%) scored higher than or equal to 32, which possibly hints at ASD (Baron-Cohen et al., 2001). We used dimensional scores for the remainder of the analyses, as these may capture more variability in the data than binary scores (see also, Goris et al., 2017). Cronbach's alpha of the AQ was 0.84, which indicates good internal consistency. The total raw score on the SRS-A ranged from 4 to 110 $(M=41.82, S D=21.83)$. Scores of 23 of $157(14.65 \%)$ participants indicated mild to moderate deficiency in social responsiveness. Six of 157 (3.82\%) participants' scores suggest severe deficiency in social responsiveness. Please note though that a diagnosis of ASD cannot be made based on questionnaires scores only. Cronbach's alpha was 0.94 for the SRS-A, indicating excellent internal consistency. As expected, there was a strong positive correlation between the total scores of the AQ and SRS-A, Pearson's $r=.69, p<.001$, which confirms the construct validity of both measures.

\section{Music preference paradigm}

Preferences. Participants preferred tone sequences of medium to high entropy (see Figure 1B). Similar to Delplanque and colleagues (2019), we used a linear mixed effects model (LME) to test the linear and quadratic effects of entropy on preferences for the first vs. second sequence, reporting the results with a type III Wald chi square test. The quadratic components 
were calculated by squaring the standardized entropy values, and were used to test an inverted Urelationship. This replicated the significant effect of both the linear, $b=0.23, \chi^{2}(1,156)=76.78$, $p<.001$, and quadratic, $b=-0.13, \chi^{2}(1,156)=66.87, p<001$, component of the first sequence, as well as the significant effect of both the linear, $b=-0.36, \chi^{2}(1,156)=130.07, p<.001$, and quadratic, $b=0.18, \chi^{2}(1,156)=111.46, p<.001$, component of the second sequence, as earlier shown by Delplanque and colleagues (2019).

\section{Correlational analyses.}

Percentage of predictable sequence chosen. There was a significant correlation between the percentage of trials in which participants preferred the most predictable sequence (i.e. with the lowest entropy) and the PCA autism factor score, $\rho=.20, p=.01$. This overall preference also showed a marginally significant correlation with the AQ total score, $\rho=.16, p=.05$, and a significant correlation with the SRS-A total score, $\rho=.23, p<.01$ (see Figure 1C). These correlations indicate a small to medium effect size according to Cohen's classical benchmarks (1988), or a medium effect size according to more recent meta-analyses (Bosco, Aguinis, Singh, Field, \& Pierce, 2015; Schäfer \& Schwarz, 2019).

Weighted sum. As an alternative measure of preference for predictability, we also calculated a weighted sum score of the preferences for the different classes as the sum of the preferences for each class, multiplied with a weight factor indicating the predictability of each class, using the following formula.

$$
\text { Weighted sum }=\sum_{i=1}^{11}(12-i) \text { preference } \text { class } i
$$


This way, higher numbers mean stronger preferences for more predictable tunes. This weighted sum again correlated significantly with the PCA autism factor score, $\rho=.19, p=.02$, as well as with the SRS-A, $\rho=.23, p<.01$, but not with the AQ, $\rho=.13, p=.11$.

A.

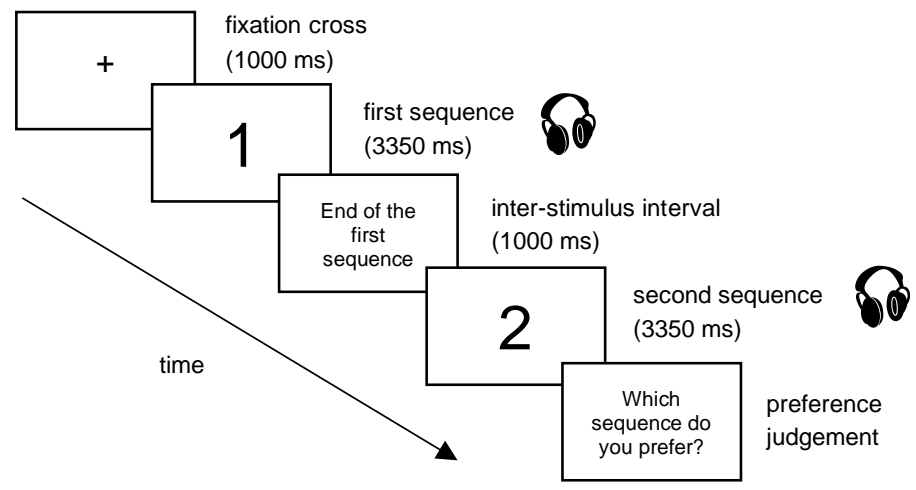

C.

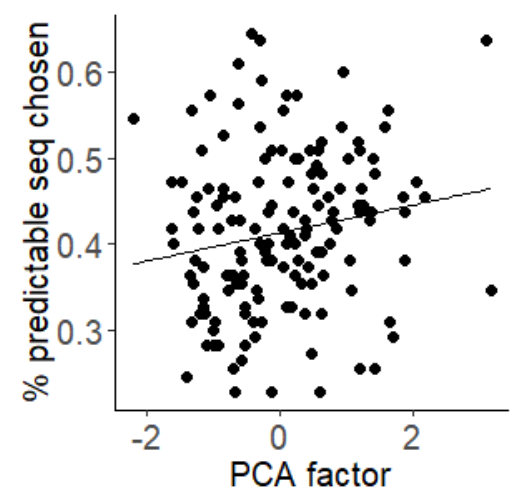

B.

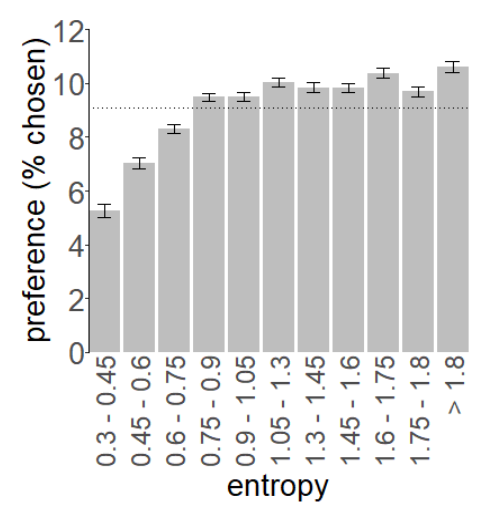

entropy

Figure 1. Paradigm and main results of the Music Preference paradigm. (A) Schematic representation of a single trial. (B) Mean percentage of trials in which sequences from different entropy classes were preferred. Error bars represent \pm 1 standard error of the mean (SEM). The dotted line reflects the chance level of $9 \%$, i.e. the percentage in which sequences from each entropy class could be chosen if participants' preferences would not be influenced by entropy. (C) Correlations between the percentage of trials in which participants preferred the most predictable sequence and Principal Components Analysis (PCA) factor, Autism Spectrum Quotient (AQ), and Social Responsiveness Scale - Adult version (SRS-A). For descriptive purposes, we plotted a trend line reflecting the Pearson correlation coefficient. 


\section{Perceptual fluency paradigm}

Preferences. In contrast to previous studies (Forster et al., 2012; Reber et al., 1998;

Westerman et al., 2015), there was no significant difference in preference for targets between congruent and incongruent trials, $t(160)=1.07, p=.29$ (see Figure $2 \mathrm{~B}$ ). To investigate whether participants did show differences in preferences across images, we looked at the individual ranges of ratings, which were between 1-7 (broadest range) and 2-5 (smallest range). This suggests that all participants sufficiently varied their responses. Furthermore, there was no significant difference in RT between congruent and incongruent trials, $t(160)=1.13, p=.26$.

Correlational analyses. Interestingly, there was a significant correlation between preference for targets in congruent vs. incongruent trials and the PCA autism factor score, $\rho=.18$, $p=.03$, as well as the AQ and SRS-A, separately, $\rho=.17, p=.03$, and $\rho=.16, p=.045$, respectively (see Figure 2C). 
A.

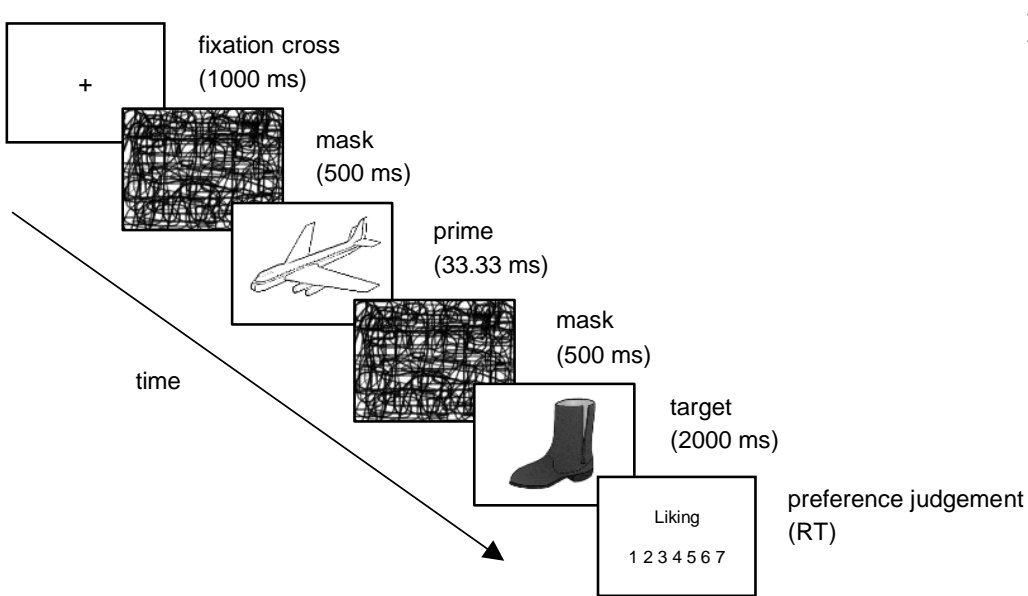

B.

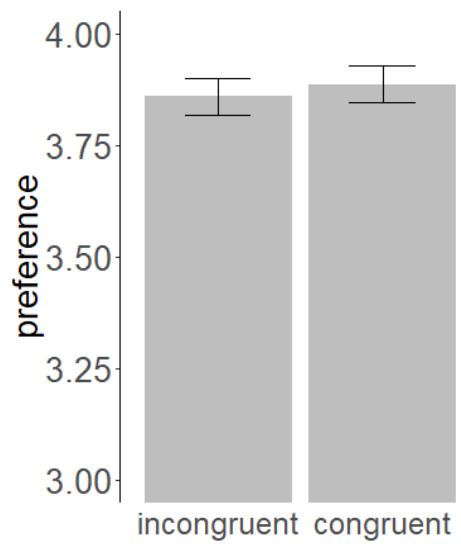

C.
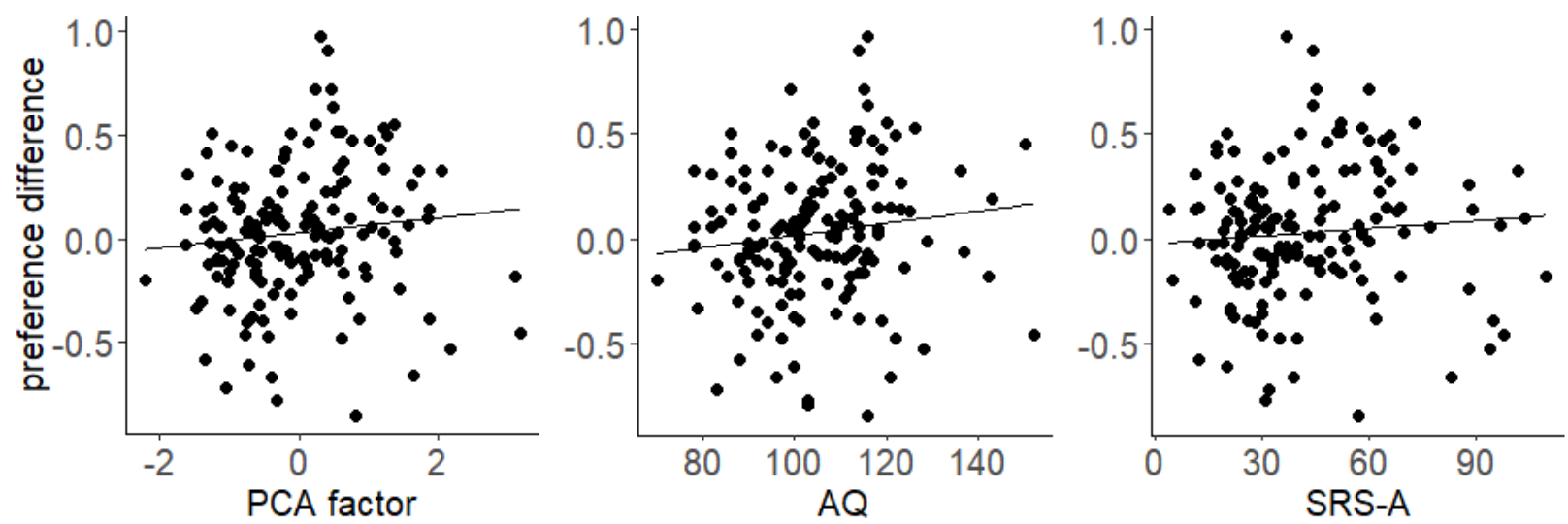

Figure 2. Paradigm and main results of the Perceptual Fluency paradigm. (A) Schematic representation of an incongruent trial. (B) Mean preference for incongruent and congruent trials. Error bars represent \pm 1 standard error of the mean (SEM). (C) Correlations between the difference in preference for congruent minus incongruent trials, and the Principal Components Analysis (PCA) factor, Autism Spectrum Quotient (AQ) and Social Responsiveness Scale - Adult version (SRS-A). For descriptive purposes, we plotted a trend line reflecting the Pearson correlation coefficient.

\section{Gambling task}

Preferences. On average, the fixed deck was chosen in $33 \%$ of trials, the SD10 deck in $20 \%$, the SD30 in $21 \%$ and the SD70 deck in 26\%, see also Figure 3B. Mean RT was $510 \mathrm{~ms}$ ( $S D=499 \mathrm{~ms}$ ). 


\section{Correlational analyses.}

Preference for the fixed deck. There were no significant correlations between preference for the fixed deck and PCA factor score, $\rho=.07, p=.38$, nor with AQ, $\rho=.04, p=.63$, or SRS-A, $\rho=.07, p=.40$.

Weighted sum score. The weighted sum score was defined as the sum of preferences for each deck multiplied with a weight indicating the predictability for each deck, so that higher numbers mean stronger preferences for predictable decks, using the following formula.

$$
\text { Weighted sum }=\sum_{i=1}^{4}(5-i) \text { preference } \text { deck } i
$$

There were no significant correlations with PCA factor score, $\rho=.01, p=.91$, nor with $\mathrm{AQ}, \rho=.01, p=.87$, or SRS-A, $\rho=.02, p=.77$.

Reaction times. As an exploratory analysis, we also investigated the RT differences between the (mean of the) fixed and the (balanced mean of the) unpredictable decks, similar to other gambling studies (e.g. Smith, Xiao, \& Bechara, 2012). This measure correlated significantly with the PCA factor score, $\rho=.18, p=.03$, the AQ, $\rho=.19, p=.02$, and marginally significantly with SRS-A, $\rho=.14, p=.09$, meaning that participants with higher ASD-like traits tend to choose more quickly for the fixed deck compared to the three other decks (see Figure 3C). RTs were not registered in the Music Preference paradigm, and did not correlate with autistic traits in the Perceptual Fluency paradigm. 
A.

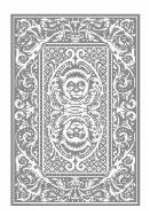

$M=€ 250$ $S D=0$

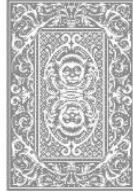

$M=€ 250$

$S D=10$

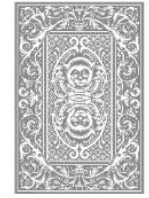

$M=€ 250$

$S D=30$

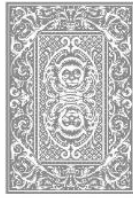

$M=€ 250$

$S D=70$
B.

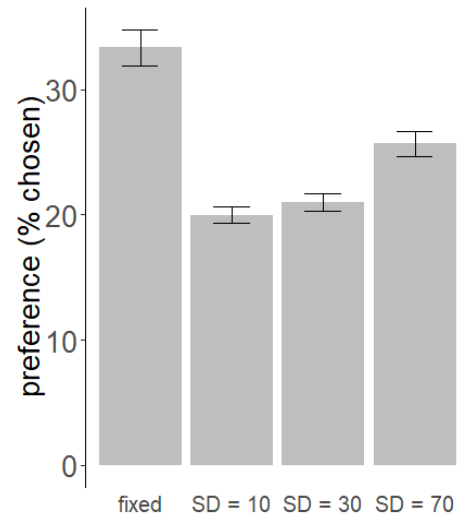

C.
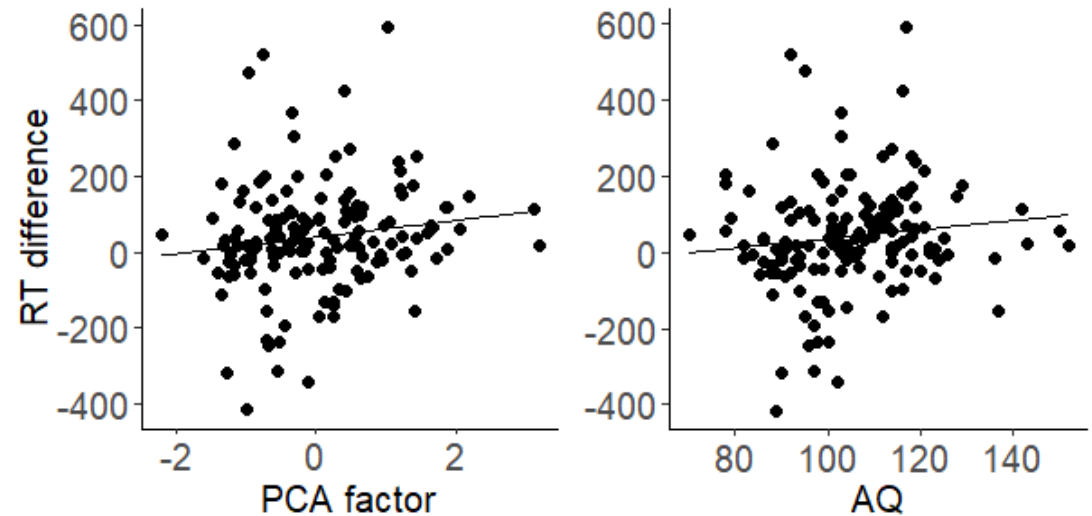

Figure 3. Paradigm and main results of the Gambling Task. (A) Schematic representation of the different decks of cards. (B) Mean preference for the different decks. Error bars represent \pm 1 standard error of the mean (SEM). (C) Correlations between the difference in reaction time (RT) between the three unpredictable decks minus the fixed deck, and the Principal Components Analysis (PCA) factor, Autism Spectrum Quotient (AQ), and Social Responsiveness Scale - Adult version (SRS-A). For descriptive purposes, we plotted a trend line reflecting the Pearson correlation coefficient. 


\section{Exploratory analyses across paradigms}

Correlational analyses across paradigms. To explore whether the relation between preference for predictability and autistic traits reflects a generalizable trait across the three different paradigms, or is more context-specific instead, we investigated the relations between the aforementioned preference measures of the different paradigms. Interestingly, there were no significant correlations, all Spearman's $|\rho|<.13$, all $p>.12$.

Correlations with the subscales. Knowing that these different measures of preference for predictability did not correlate with one another, we further investigated whether this meant that they were also associated to different subscales of the AQ and SRS-A. Interestingly, as indicated in Table 1, although all three measures of predictability showed a positive relation with the total scores, different measures seem to be related to different subscales.

Table 1. Spearman's rank-ordered coefficients $\rho$ for the correlations between the different task measures and the subscales of the Autism Spectrum Quotient (AQ) and the Social Responsiveness Scale - Adult version (SRS-A). The full names of the subscales can be found in the methods section. Please note that the significance levels are not corrected for multiple comparisons: $* p<0.05$, ** $p<0.01$.

\begin{tabular}{lccccccccc}
\hline & $\begin{array}{c}\text { AQ } \\
\text { social }\end{array}$ & $\begin{array}{c}\text { AQ } \\
\text { switch }\end{array}$ & $\begin{array}{c}\text { AQ } \\
\text { detail }\end{array}$ & $\begin{array}{c}\text { AQ } \\
\text { comm }\end{array}$ & $\begin{array}{c}\text { AQ } \\
\text { imag }\end{array}$ & $\begin{array}{c}\text { SRS-A } \\
\text { consc }\end{array}$ & $\begin{array}{c}\text { SRS-A } \\
\text { comm }\end{array}$ & $\begin{array}{c}\text { SRS-A } \\
\text { motiv }\end{array}$ & $\begin{array}{c}\text { SRS-A } \\
\text { rigid }\end{array}$ \\
\hline $\begin{array}{l}\text { Music Preference: } \\
\text { \% predictable chosen }\end{array}$ & 0.10 & 0.00 & -0.02 & $\mathbf{0 . 2 6 * *}$ & 0.11 & $\mathbf{0 . 2 1} * *$ & $\mathbf{0 . 2 2} * *$ & 0.15 & $\mathbf{0 . 2 0 *}$ \\
$\begin{array}{l}\text { Perceptual Fluency: } \\
\text { preference difference }\end{array}$ & $\mathbf{0 . 1 7 *}$ & 0.10 & 0.07 & 0.11 & 0.06 & 0.04 & 0.15 & $\mathbf{0 . 2 4 * *}$ & 0.07 \\
$\begin{array}{l}\text { Gambling: } \\
\text { RT difference }\end{array}$ & 0.12 & 0.14 & $\mathbf{0 . 1 9 *}$ & 0.00 & 0.06 & 0.11 & 0.13 & 0.13 & $\mathbf{0 . 1 7 ^ { * }}$ \\
\hline
\end{tabular}




\section{Discussion}

While an above average preference for predictability or sameness is one of the main symptoms of ASD, it has received surprisingly little attention in research. Several studies have shown that a preference for predictability is present in the neurotypical population (Chetverikov \& Kristjansson, 2015; Delplanque et al., 2019; Forster et al., 2012; Ogawa \& Watanabe, 2011; Trapp et al., 2015; Westerman et al., 2015), but the association with ASD has rarely been studied and a positive relation has not yet been shown. In fact, in a previous attempt to investigate this, we did not find any relation with autistic traits (Goris et al., 2017). In the current study, we used three different paradigms measuring preferences for predictability and correlated them with autistic traits in a large neurotypical sample.

Our findings show that autistic traits indeed correlate significantly with preferences for predictable tone sequences and images that were preceded by a similar subliminal prime. In contrast to our hypothesis, there was no correlation with preferring the predictable outcome in a gambling task, which could be due to other factors confounding the predictability manipulation such as reward processing and risk taking, which might also be different in ASD (Faja, Murias, Beauchaine, \& Dawson, 2013; Mussey, Travers, Klinger, \& Klinger, 2015). However, we did find a post-hoc correlation with RT, which could indicate that participants with more autistic traits have an implicit preference for the deck with a predictable outcome, as they were faster in choosing this deck. While further research in clinical samples is necessary, these results are the first to show that a relation between preference for predictability and autistic traits can be observed in a standardized lab setting. As indicated in the introduction, the reason we could not observe this earlier (Goris et al., 2017) might lie in the fact that we made participants aware of 
the predictability manipulation in our previous study, and used a separate learning and preference phase (which required a transfer of preferences across contexts).

Surprisingly, the different task measures did not correlate with each other and seemed related to different symptoms of ASD (as measured with different subscales of the AQ and SRSA). This could suggest that preference for predictability does not reflect a single domain-general process in ASD, but rather that this is a context-sensitive (coping) mechanism that can manifest itself differently depending on the nature and the severity of different autistic traits. These observations seem consistent with what is known about Intolerance of Uncertainty (IU), which has also been shown to be a multidimensional construct (Boulter, Freeston, South, \& Rodgers, 2014; Carleton et al., 2012). IU is a dispositional risk factor for developing anxiety, usually investigated using questionnaires in depression and anxiety disorders (Carleton et al., 2012; for a review, see Einstein, 2014), and ASD (Boulter et al., 2014; Neil et al., 2016; Wigham et al., 2015). While the relationship between IU and the current paradigms measuring preference for predictability is unclear and should be investigated further, it is possible that the different tasks are also related to different factors of IU.

Notably, the subscale analyses indicated no relations with the attention switching subscale in the AQ and only a relation with the subscale rigidity and repetitiveness in the SRS-A for two out of three paradigms. This is interesting because these subscales are thought to measure insistence on sameness and preference for routines. The fact that we found stronger relations with some of the other, more socially oriented subscales could suggest that preference for predictability as measured in our tasks correlates with a broader spectrum of autistic traits, and not with self-reported preferences for routines alone. However, we believe caution is warranted regarding the interpretation of these subscale analyses, as these were only exploratory, post-hoc 
analyses and we did not have strong hypotheses about them. Our results are also interesting in the light of recent theories that emphasized problems in dealing with unpredictability in ASD (Lawson et al., 2014; Pellicano \& Burr, 2012; Van de Cruys et al., 2014; Van de Cruys, Van der Hallen, \& Wagemans, 2017). Based on the predictive coding framework, it is proposed that a deficit in processing unexpected information might be the core deficit accounting for all ASD symptoms (for an overview, see Palmer et al., 2017). Specifically, these theories suggest that there would be a problem with the context-sensitive adjustment of prediction errors (i.e. surprising events) in ASD. This would lead to an overload of surprises in the autistic brain, which on its turn explains the preference for sameness and predictability in ASD, among other symptoms. Indeed, some studies have provided first support for this hypothesis, such as a weaker context modulation of neurophysiological prediction error processing (Goris et al., 2018) and atypical surprise responses (Lawson et al., 2017). More generally, these theories treat the aberrant processing of (un)predictable information in ASD more as an explanans rather than an explanandum, and invite us to investigate this core symptom of ASD more systematically. In this respect, the present study should be seen as an important first step in bringing this symptom closer to the lab, which on its turn will allow for a more controlled investigation of its underlying (neurophysiological) mechanisms. Moreover, by testing this symptom under controlled experimental conditions, we also demonstrated how this symptom can be generalized to these new conditions. Specifically, when describing insistence on sameness in ASD, psychologists typically refer to expectations that developed over relatively long timescales (e.g., daily routines), while the preferences tested here relate to a preference for predictability over very short timescales (e.g. within a few seconds in the Music Preference and Perceptual Fluency paradigm). The fact that we still found correlations with these task measures, suggests that the 
preference for predictability in ASD is not restricted to a preference for routines and sameness in daily life, but also encompasses preferences for low-level short-term predictability in sensory input, which is consistent with predictive coding accounts of ASD.

Interestingly, some studies have suggested that insistence on sameness could be a mechanism to cope with anxiety or distress (Boulter et al., 2014; Rodgers, Glod, Connolly, \& McConachie, 2012; Spiker et al., 2012). Here, we demonstrate that insistence of sameness can also be observed at a much more basic level, and could thus reflect a more inherent property of information processing in autism. Either way, it would certainly be interesting to investigate how the measures of preferences for sameness and predictability are modulated by (social) stress.

In conclusion, we have shown in three different paradigms that the relation between preference for predictability and autistic traits can be observed under well-controlled experimental conditions. Surprisingly, the task measures of the different paradigms did not correlate with each other and were related to different symptoms of ASD. As insistence on sameness and predictability is a well-known but poorly understood symptom of ASD, we hope that the current findings will contribute to a deeper understanding of its underlying mechanisms and spark interest for future systematic investigations. 


\section{References}

American Psychiatric Association (2013). Diagnostic and statistical manual of mental disorders, 5th ed. Arlington, VA: American Psychiatric Publishing.

Ashwood, K. L., Gillan, N., Horder, J., Hayward, H., Woodhouse, E., McEwen, F. S., ... Murphy, D. G. (2016). Predicting the diagnosis of autism in adults using the AutismSpectrum Quotient (AQ) questionnaire. Psychological Medicine, 46(12), 2595-2604. https://doi.org/10.1017/S0033291716001082

Baron-Cohen, S., Wheelwright, S., Skinner, R., Martin, J., \& Clubley, E. (2001). The autismspectrum quotient (AQ): evidence from Asperger syndrome/high- functioning autism, males and females, scientists and mathematicians. Journal of Autism and Developmental Disorders, 31(1), 5-17. https://doi.org/10.1023/A:1005653411471

Bechara, A., Damasio, A. R., Damasio, H., \& Anderson, S. W. (1994). Insensitivity to future consequences following damage to human prefrontal cortex. Cognition, 50(1-3), 7-15. https://doi.org/10.1016/0010-0277(94)90018-3

Bechara, A., Damasio, H., Tranel, D., \& Damasio, A. R. (2005). The Iowa Gambling Task and the somatic marker hypothesis: some questions and answers. Trends in Cognitive Sciences, 9(4), 159-162. https://doi.org/10.1016/J.TICS.2005.02.002

Bosco, F. A., Aguinis, H., Singh, K., Field, J. G., \& Pierce, C. A. (2015). Correlational Effect Size Benchmarks. Journal of Applied Psychology, 100(2), 431-449. Retrieved from http://www.hermanaguinis.com/JAP2015.pdf

Boulter, C., Freeston, M., South, M., \& Rodgers, J. (2014). Intolerance of Uncertainty as a 
Framework for Understanding Anxiety in Children and Adolescents with Autism Spectrum Disorders. Journal of Autism and Developmental Disorders, 44(6), 1391-1402. https://doi.org/10.1007/s10803-013-2001-X

Carleton, R. N., Mulvogue, M. K., Thibodeau, M. A., McCabe, R. E., Antony, M. M., \& Asmundson, G. J. G. (2012). Increasingly certain about uncertainty: Intolerance of uncertainty across anxiety and depression. Journal of Anxiety Disorders, 26(3), 468-479. https://doi.org/10.1016/J.JANXDIS.2012.01.011

Chetverikov, A., \& Kristjansson, Á. (2015). History effects in visual search for monsters: Search times, choice biases, and liking. Attention, Perception, \& Psychophysics, 77(2), 402-412. https://doi.org/10.3758/s13414-014-0782-4

Constantino, J. N., Davis, S. A., Todd, R. D., Schindler, M. K., Gross, M. M., Brophy, S. L., et al. (2003). Validation of a brief quantitative measure of autistic traits: Comparison of the social responsiveness scale with the autism diagnostic interview-revised. Journal of Autism and Developmental Disorders, 33(4), 427-433. https://doi.org/10.1023/A:1025014929212

Constantino, J. N., \& Todd, R. D. (2003). Autistic Traits in the General Population. Archives of General Psychiatry, 60(5), 524. https://doi.org/10.1001/archpsyc.60.5.524

Delplanque, J., De Loof, E., Janssens, C., \& Verguts, T. (2019). The sound of beauty: How complexity determines aesthetic preference. Acta Psychologica, 192, 146-152. https://doi.org/10.1016/J.ACTPSY.2018.11.011

Dreisbach, G., \& Fischer, R. (2012). Conflicts as aversive signals. Brain and Cognition, 78(2), 94-98. https://doi.org/10.1016/J.BANDC.2011.12.003 
Einstein, D. A. (2014). Extension of the Transdiagnostic Model to Focus on Intolerance of Uncertainty: A Review of the Literature and Implications for Treatment. Clinical Psychology: Science and Practice, 21(3), 280-300. https://doi.org/10.1111/cpsp.12077

Faja, S., Murias, M., Beauchaine, T. P., \& Dawson, G. (2013). Reward-Based Decision Making and Electrodermal Responding by Young Children with Autism Spectrum Disorders during a Gambling Task. Autism Research, 6(6), 494-505. https://doi.org/10.1002/aur.1307

Forster, M., Leder, H., \& Ansorge, U. (2012). It felt fluent, and I liked it: Subjective feeling of fluency rather than objective fluency determines liking. Emotion, 13(2), 280-289. https://doi.org/10.1037/a0030115

Fritz, J., \& Dreisbach, G. (2013). Conflicts as aversive signals: Conflict priming increases negative judgments for neutral stimuli. Cognitive, Affective, \& Behavioral Neuroscience, 13(2), 311-317. https://doi.org/10.3758/s13415-012-0147-1

Goris, J., Braem, S., Nijhof, A. D., Rigoni, D., Deschrijver, E., Van de Cruys, S., ... Brass, M. (2018). Sensory prediction errors are less modulated by global context in autism spectrum disorder. Biological Psychiatry: Cognitive Neuroscience and Neuroimaging, 3(8), 667-674. https://doi.org/10.1016/j.bpsc.2018.02.003

Goris, J., Deschrijver, E., Trapp, S., Brass, M., \& Braem, S. (2017). Autistic traits in the general population do not correlate with a preference for associative information. Research in Autism Spectrum Disorders, 33, 29-38. https://doi.org/10.1016/j.rasd.2016.11.001

Grinter, E. J., Maybery, M. T., Van Beek, P. L., Pellicano, E., Badcock, J. C., \& Badcock, D. R. (2009). Global Visual Processing and Self-Rated Autistic-like Traits. Journal of Autism and Developmental Disorders, 39(9), 1278-1290. https://doi.org/10.1007/s10803-009-0740-5 
Hoekstra, R., Bartels, M., Cath, D., \& Boomsma, D. (2008). Factor structure, reliability and criterion validity of the autism-spectrum quotient (AQ): A study in Dutch population and patient groups. Journal of Autism and Developmental Disorders, 38, 1555-1566. https://doi.org/10.1007/s10803-008-0538-X

Ingersoll, B., Hopwood, C. J., Wainer, A., \& Brent Donnellan, M. (2011). A Comparison of Three Self-Report Measures of the Broader Autism Phenotype in a Non-Clinical Sample. Journal of Autism and Developmental Disorders, 41(12), 1646-1657. https://doi.org/10.1007/s10803-011-1192-2

Landry, O., \& Al-Taie, S. (2016). A Meta-analysis of the Wisconsin Card Sort Task in Autism. Journal of Autism and Developmental Disorders, 46(4), 1220-1235. https://doi.org/10.1007/s10803-015-2659-3

Lawson, R. P., Mathys, C., \& Rees, G. (2017). Adults with autism overestimate the volatility of the sensory environment. Nature Neuroscience, 20(9), 1293-1299. https://doi.org/10.1038/nn.4615

Lawson, R. P., Rees, G., \& Friston, K. J. (2014). An aberrant precision account of autism. Frontiers in Human Neuroscience, 8, 302. https://doi.org/10.3389/fnhum.2014.00302

Mussey, J. L., Travers, B. G., Klinger, L. G., \& Klinger, M. R. (2015). Decision-Making Skills in ASD: Performance on the Iowa Gambling Task. Autism Research, 8(1), 105-114. https://doi.org/10.1002/aur.1429

Neil, L., Olsson, N. C., \& Pellicano, E. (2016). The Relationship Between Intolerance of Uncertainty, Sensory Sensitivities, and Anxiety in Autistic and Typically Developing Children. Journal of Autism and Developmental Disorders, 46(6), 1962-1973. 
https://doi.org/10.1007/s10803-016-2721-9

Noens, I., De la Marche, W., \& Scholte, E. (2012). Screeningslijst voor autismespectrumstoornissen bij volwassenen. Amsterdam: Hogrefe Uitgevers B.V..

Ogawa, H., \& Watanabe, K. (2011). Implicit learning increases preference for predictive visual display. Attention, Perception \& Psychophysics, 73(6), 1815-1822. https://doi.org/10.3758/s13414-010-0041-2

Palmer, C. J., Lawson, R. P., \& Hohwy, J. (2017). Bayesian Approaches to Autism: Towards Volatility, Action, and Behavior. Psychological Bulletin, 143(5), 521-542. https://doi.org/10.1037/bul0000097

Pellicano, E., \& Burr, D. (2012). When the world becomes "too real": A Bayesian explanation of autistic perception. Trends in Cognitive Sciences, 16(10), 504-510. https://doi.org/10.1016/j.tics.2012.08.009

Poljac, E., Poljac, E., \& Yeung, N. (2012). Cognitive control of intentions for voluntary actions in individuals with a high level of autistic traits. Journal of Autism and Developmental Disorders, 42(12), 2523-2533. https://doi.org/10.1007/s10803-012-1509-9

Reber, R., Winkielman, P., \& Schwarz, N. (1998). Effects of Perceptual Fluency on Affective Judgments. Psychological Science, 9(1), 45-48. https://doi.org/10.1111/1467-9280.00008

Robertson, A. E., \& Simmons, D. R. (2013). The Relationship between Sensory Sensitivity and Autistic Traits in the General Population. Journal of Autism and Developmental Disorders, 43(4), 775-784. https://doi.org/10.1007/s10803-012-1608-7

Rodgers, J., Glod, M., Connolly, B., \& McConachie, H. (2012). The Relationship Between 
Anxiety and Repetitive Behaviours in Autism Spectrum Disorder. Journal of Autism and Developmental Disorders, 42(11), 2404-2409. https://doi.org/10.1007/s10803-012-1531-y

Rossion, B., \& Pourtois, G. (2004). Revisiting Snodgrass and Vanderwart's Object Pictorial Set: The Role of Surface Detail in Basic-Level Object Recognition. Perception, 33(2), 217-236. https://doi.org/10.1068/p5117

Schäfer, T., \& Schwarz, M. A. (2019). The Meaningfulness of Effect Sizes in Psychological Research: Differences Between Sub-Disciplines and the Impact of Potential Biases. Frontiers in Psychology, 10, 813. https://doi.org/10.3389/fpsyg.2019.00813

Schouppe, N., Braem, S., De Houwer, J., Silvetti, M., Verguts, T., Ridderinkhof, K. R., \& Notebaert, W. (2015). No pain, no gain: the affective valence of congruency conditions changes following a successful response. Cognitive, Affective, \& Behavioral Neuroscience, 15(1), 251-261. https://doi.org/10.3758/s13415-014-0318-3

Smith, D. G., Xiao, L., \& Bechara, A. (2012). Decision making in children and adolescents: Impaired Iowa Gambling Task performance in early adolescence. Developmental Psychology, 48(4), 1180-1187. https://doi.org/10.1037/a0026342

South, M., Chamberlain, P. D., Wigham, S., Newton, T., Le Couteur, A., McConachie, H., ... Rodgers, J. (2014). Enhanced decision making and risk avoidance in high-functioning autism spectrum disorder. Neuropsychology, 28(2), 222-228. https://doi.org/10.1037/neu0000016

Spiker, M. A., Lin, C. E., Van Dyke, M., Wood, J. J., Dyke, M. Van, \& Wood, J. J. (2012). Restricted interests and anxiety in children with autism. Autism, 16(3), 306-320. https://doi.org/10.1177/1362361311401763 
Stewart, M. E., Watson, J., Allcock, A.-J., \& Yaqoob, T. (2009). Autistic traits predict performance on the block design. Autism, 13(2), 133-142. https://doi.org/10.1177/1362361308098515

Trapp, S., Shenhav, A., Bitzer, S., \& Bar, M. (2015). Human preferences are biased towards associative information. Cognition \& Emotion, 29(6), 1054-1068. https://doi.org/10.1080/02699931.2014.966064

Tulver, K., Aru, J., Rutiku, R., \& Bachmann, T. (2019). Individual differences in the effects of priors on perception: a multi-paradigm approach. Cognition, 187, 167-177. https://doi.org/10.1101/523324

Van de Cruys, S., Evers, K., Van der Hallen, R., Van Eylen, L., Boets, B., De-Wit, L., \& Wagemans, J. (2014). Precise minds in uncertain worlds: predictive coding in autism. Psychological Review, 121(4), 649-675. https://doi.org/10.1037/a0037665

Van de Cruys, S., Van der Hallen, R., \& Wagemans, J. (2017). Disentangling signal and noise in autism spectrum disorder. Brain and Cognition, 112, 78-83. https://doi.org/10.1016/j.bandc.2016.08.004

Walter, E., Dassonville, P., \& Bochsler, T. M. (2009). A Specific Autistic Trait that Modulates Visuospatial Illusion Susceptibility. Journal of Autism and Developmental Disorders, 39(2), 339-349. https://doi.org/10.1007/s10803-008-0630-2

Westerman, D. L., Lanska, M., \& Olds, J. M. (2015). The effect of processing fluency on impressions of familiarity and liking. Journal of Experimental Psychology: Learning, Memory, and Cognition, 41(2), 426-438. https://doi.org/10.1037/a0038356 
White, S. W., Ollendick, T. H., \& Bray, B. C. (2011). College students on the autism spectrum: Prevalence and associated problems. Autism, 15(6), 683-701. https://doi.org/10.1177/1362361310393363

Wigham, S., Rodgers, J., South, M., McConachie, H., \& Freeston, M. (2015). The Interplay Between Sensory Processing Abnormalities, Intolerance of Uncertainty, Anxiety and Restricted and Repetitive Behaviours in Autism Spectrum Disorder. Journal of Autism and Developmental Disorders, 45(4), 943-952. https://doi.org/10.1007/s10803-014-2248-x

Woodbury-Smith, M. R., Robinson, J., Wheelwright, S., \& Baron-Cohen, S. (2005). Screening adults for Asperger Syndrome using the AQ: A preliminary study of its diagnostic validity in clinical practice. Journal of Autism and Developmental Disorders, 35(3), 331-335. https://doi.org/10.1007/s10803-005-3300-7

Zajonc, R. B. (2001). Mere Exposure: A Gateway to the Subliminal. Current Directions in Psychological Science, 10(6), 224-228. https://doi.org/10.1111/1467-8721.00154 